\title{
CORRESPONDENCE
}

\section{Involvement of pharmacies in tuberculosis treatment}

\section{To the Editors:}

In a recent issue of the European Respiratory Journal, Yew et al. [1] recalled the change from DOTS (directly observed treatment, short course) strategy to a more comprehensive approach, the Stop TB Strategy, adopted by the World Health Organization. Three of the six components of this strategy are the pursuit of high-quality DOTS expansion and enhancement, the engagement of all care providers, and the empowerment of people with tuberculosis and communities through partnership. We present a project based on the decentralisation of treatment and on patient participation in the choice of DOT supporter, in line with the Stop TB Strategy as presented by YEW et al. [1]. In Portugal, DOT is mandatory, and is performed principally in an outpatient setting within Pulmonary Health Centers (CDPs); these are primary care units dedicated to the diagnosis, treatment and surveillance of tuberculosis. Tuberculosis patients continue to suffer from social stigma and find it embarrassing to be subjected to DOT in CDP facilities. To deal with this problem, the Vila Nova de Gaia CDP team and the National Association of Pharmacies (ANF) designed and implemented a 6-month pilot programme permitting DOT in community pharmacies of the county of Vila Nova de Gaia. The aim was to assess the feasibility of such an innovation and its effect on patient outcomes.

The CDP medical team defined the inclusion criteria: all new cases of fully drug-susceptible tuberculosis [2] in the continuation phase of treatment and expressing displeasure at being forced to receive treatment at the CDP were eligible. The ANF conducted a survey of pharmacies in Vila Nova de Gaia county to identify those with the physical infrastructure, the staff capacity and the willingness to participate in the programme. No reward was offered, the gain to the pharmacies being a strengthened relationship with patients from a community with possible commercial benefits. The pharmacists were trained by CDP doctors. Training included a discussion of tuberculosis epidemiology, the immunopathogenesis of Mycobacterium tuberculosis, and tuberculosis diagnosis and treatment. Within this overall theme, the concept of DOT and the rules for effective DOT were defined. An administration guidebook was prepared. This functioned simultaneously to assist in anti-tuberculosis drug prescription as well as to provide indicators of compliance with therapy. Selected patients were invited to participate in DOT at a pharmacy near their homes. No patient was obliged to accept the treatment transfer. After patient agreement was secured, the local pharmacist was contacted to determine the day on which DOT would commence. The drug administration guidebook and the anti-tuberculosis drugs were delivered to the pharmacy 2 days prior to the first local DOT. Telephone contact was established between each pharmacy and each patient's physician to ensure rapid resolution of any problematic situations, including treatment fault. In such an instance, it was agreed that the patient would be summoned to the CDP. Monthly meetings were conducted between the CDP team, the ANF delegate and participating pharmacists to monitor the situation of all patients and to evaluate programme success. All participating patients were asked to complete a questionnaire assessing their satisfaction at day 30 and at the end of the treatment. Patients continued to receive monthly consultations with their CDP doctors. To assess programme success, the outcomes of all patients were noted and compared with those of outpatients undergoing DOT in CDPs nationwide.

12 local pharmacies joined the programme. From June to December 2009, the programme was offered to 14 patients who fulfilled the inclusion criteria. There were eight males and six females, all new cases and none of them were immigrants or HIV positive. All of them had completed 2 months of an isoniazid $(\mathrm{H})$, rifampicin $(\mathrm{R})$, pyrazinamide and ethambutol regimen at the $\mathrm{CDP}$ (achieving negative smear and culture), and were initiating 4 months of HR at the time of transfer. The responses to the questionnaires completed on day 30 and at the end of treatment revealed high levels of satisfaction from all patients. During the 6 months of the programme, no anomalous situation with respect to DOT was observed and no faults in the treatment occurred. The operation of the pharmacies continued as normal. All patients fully completed treatment with $100 \%$ cure [2]. No tuberculosis relapse was noted upon follow-up of the 14 patients.

In 2009, the cure rate of tuberculosis patients receiving DOT in CDPs nationwide was $87 \%$ [3]. Our cure rate was $100 \%$. In terms of sample size, the probability of such an outcome was only $14.23 \%\left(0.87^{14}=0.1423\right)$ if it is assumed that the probability that our patients would receive effective treatment was the same as that of tuberculosis patients treated at CDPs. Despite our small sample size, the result suggests an improvement in the treatment outcome's prospects with our model.

The use of a facility other than a CDP by tuberculosis patients embarrassed by the social stigma associated with everyday visits to a "known tuberculosis centre" promotes adherence by tailoring DOT to the patient needs.

The limitations of our study are that we compared our results with nationwide data and did not randomise study groups. However, as we sought to develop an alternative DOT model to assist patients at risk of nonadherence because of embarrassment associated with visiting the CDP facility, we considered it unethical to randomise disaffected patients; this would have increased the risk of nonadherence in patients assigned to continue CDP-based DOT.

Thus, we formed a public-private partnership to develop a DOT programme serving patient needs, and observed $100 \%$ adherence and cure. These results confirm earlier findings on the utility of the participation of pharmacies in TB treatment [4] in 
agreement with the principles of the new European Union Standards for Tuberculosis Care [5].

Ana Antunes*, Joana Gomes*, Inês Belchior ${ }^{\#}$, Ana Isabel Loureiro", Aurora Carvalho ${ }^{*,+}$, Anabela Madeira ${ }^{\S}$ and Raquel Duarte $^{*,+, f, * *}$

${ }^{*}$ Centro Hospitalar Vila Nova de Gaia/Espinho, EPE, ${ }^{+} \mathrm{CDP}$ de Vila Nova de Gaia, Vila Nova de Gaia, "Hospital Sao Joao, EPE, ${ }^{f}$ Centro de Referência Regional do Norte de Tuberculose Multirresistente, ${ }^{* *}$ Faculdade de Medicina da Universidade do Porto, Porto, "Centro Hospitalar Trás-os-Montes e Alto Douro, Vila Real, and ${ }^{\S}$ Associação Nacional de Farmácias, Lisbon, Portugal.

Correspondence: A. Antunes, Centro Hospitalar Vila Nova de Gaia/Espinho, EPE, R Conceiçao Fernandes, Vila Nova de Gaia, Portugal. E-mail: ana.oliveirinha2@gmail.com
Statement of Interest: None declared.

\section{REFERENCES}

1 Yew WW, Lange C, Leung CC. Treatment of tuberculosis: update 2010. Eur Respir J 2011; 37: 441-462.

2 World Health Organization. Treatment of tuberculosis - guidelines for national programmes, 4th Edn. Geneva, World Health Organization, 2009.

3 ARS Norte. Congresso Internacional de Cuidados Paliativos. [International Congress on Palliative Care.] www.arsnorte.min-saude. pt/portal/page/portal/ARSNorte Date last accessed: April 30, 2011.

4 Juan $G$, Lloret $T$, Perez $C$, et al. Directly observed treatment for tuberculosis in pharmacies compared with self-administered therapy in Spain. Int J Tuberc Lung Dis 2006; 10: 215-221.

5 Migliori GB, Zellweger JP, Abubakar I, et al. European Union Standards for Tuberculosis Care. Eur Respir J 2012; 39: 807-819.

DOI: $10.1183 / 09031936.00005012$

\section{Predicting survival in mesothelioma}

\section{From the author:}

Thank you for the opportunity to respond to the thoughtful letter from J.G. Smith [1].

The main issues she raises stem from the original paper having aims other than use for prediction in current individual or legal cases. Our main aim was to describe the epidemiology of malignant mesothelioma in Western Australia and to demonstrate how little improvement there has been in survival over the last $30 \mathrm{yrs}$, despite large changes in treatment [2]. Thus, we fitted survival models to all available data. The complicated age terms were just the best-fitting fractional polynomial fit to these data. Further analyses revealed that the $\mathrm{u}$-shaped survival with age was caused by the comparatively poor survival in patients diagnosed under the age of $40 \mathrm{yrs}$, such that when these were excluded from analysis, a linear decline in age was the best fit. We also included all patients with an unknown site of mesothelioma, leading to the apparently anomalous better survival in this group.

We suggest that if a survival model is to be developed and used for individual prediction (possibly in legal cases), we would need to exclude: patients who were diagnosed at death; possibly all those with unrecorded histology; all those with unspecified site of disease; and possibly all those diagnosed before 1980 (or maybe 1990 or later).
To that end, we have spoken with J.G. Smith and suggested that we work together with the necessary subset of the data (which may soon include an additional year of accrued cases) in order to produce a revised publishable model that would have much greater utility in the context for which she wishes to use it.

\section{Arthur William Musk*,\#}

*Dept of Respiratory Medicine, Sir Charles Gairdner Hospital, Nedlands, and "Medicine and Population Health, University of Western Australia, Crawley, Australia.

Correspondence: A.W. Musk, Department of Respiratory Medicine, Sir Charles Gairdner Hospital, Nedlands WA 6009, Australia. E-mail: Bill.Musk@health.wa.gov.au

Statement of Interest: None declared.

\section{REFERENCES}

1 Smith JG. Predicting survival in malignant mesothelioma. Eur Respir J 2012; 40: 799.

2 Musk AW, Olsen N, Alfonso $\mathrm{H}$, et al. Predicting survival in malignant mesothelioma. Eur Respir J 2011; 38: 1420-1424.

DOI: $10.1183 / 09031936.00102612$ 